\title{
LMI-Based Stability Criterion for Impulsive Delays Markovian Jumping Time-Delays Reaction-Diffusion BAM Neural Networks via Gronwall-Bellman-Type Impulsive Integral Inequality
}

\author{
Ruofeng Rao, ${ }^{1,2}$ Xiongrui Wang, ${ }^{1}$ and Shouming Zhong ${ }^{3}$ \\ ${ }^{1}$ Department of Mathematics, Chengdu Normal University, Chengdu, Sichuan 611130, China \\ ${ }^{2}$ Institution of Mathematics, Yibin University, Yibin, Sichuan 644007, China \\ ${ }^{3}$ School of Science Mathematics, University of Electronic Science and Technology of China, Chengdu 610054, China \\ Correspondence should be addressed to Ruofeng Rao; ruofengrao@163.com
}

Received 2 February 2015; Revised 23 June 2015; Accepted 25 June 2015

Academic Editor: Asier Ibeas

Copyright ( 2015 Ruofeng Rao et al. This is an open access article distributed under the Creative Commons Attribution License, which permits unrestricted use, distribution, and reproduction in any medium, provided the original work is properly cited.

\begin{abstract}
Lyapunov stability theory, variational methods, Gronwall-Bellman-type inequalities theorem, and linear matrices inequality (LMI) technique are synthetically employed to obtain the LMI-based global stochastic exponential stability criterion for a class of timedelays Laplace diffusion stochastic equations with large impulsive range under Dirichlet boundary value, whose backgrounds of physics and engineering are the impulsive Markovian jumping time-delays reaction-diffusion BAM neural networks. As far as the authors know, it is the first time to derive the LMI-based criterion by way of Gronwall-Bellman-type inequalities, which can be easily and efficiently computed by computer Matlab LMI toolbox. And the obtained criterion improves the allowable upper bounds of impulse against those of some previous related literature. Moreover, a numerical example is presented to illustrate the effectiveness of the proposed methods.
\end{abstract}

\section{Introduction}

In this paper, we consider the stability of a class of time-delays Laplace diffusion stochastic equations with large impulsive range under Dirichlet boundary value, whose backgrounds of physics and engineering are the impulsive Markovian jumping time-delays reaction-diffusion bidirectional associative memory (BAM) neural networks. In 1987, Kosko [1] introduced originally the BAM neural networks model. Owing to its generalization of the single-layer autoassociative Hebbian correlation to two-layer pattern-matched heteroassociative circuits, the BAM neural networks have been proved to have widespread applications in many areas, such as pattern recognition, automatic control, signal and image processing, artificial intelligence, and parallel computation and optimization problems. Generally, an important precondition of the applications mentioned above is that the equilibrium of the BAM neural networks should be stable to some extent. So the stability analysis for neural networks has been attracting wide publicity ([2-6] and their references therein).
In the real world, the neural networks are often disturbed by environmental noise. The noise may influence the stability of the equilibrium and vary some structure parameters, which usually satisfies the Markov process [7-14]. In addition, diffusion effect exists really in the neural networks when electrons are moving in asymmetric electromagnetic fields. Thereby, reaction-diffusion factor should be considered in any neural networks model [2, 4-7, 15-17]. Besides, time-delays also occur unavoidably owing to the finite switching speed of neurons and amplifiers. On the other hand, impulsive effect inevitably exists in the practical neural networks, affects dynamical behaviors of the systems, and even influences the stability. And hence, the stability of impulsive neural networks was widely investigated $[5,6,10,15,16,18]$. However, there exist some harsh conditions on the impulsive range. For example, in [10, Theorem 3.1], [9, Theorem 1], [15, Theorem 1], [3, Theorem 4.1], [4, Theorem 3.2], and [19, Theorem 3.2], their impulsive condition is similar to

$$
C_{k r}^{T} P_{i} C_{k r}<P_{l} \text {. }
$$


Recently, Gronwall inequalities, Gronwall-Bellman-type inequalities, and their applications have attracted abundant interests [16, 20-23]. In this paper, we will synthetically employ Lyapunov stability theory, variational methods, Gronwall-Bellman-type inequalities theorem, and linear matrices inequality (LMI) technique to derive the LMI-based global stochastic exponential stability criterion for Markovian jumping time-delays reaction-diffusion BAM neural networks with large impulsive range allowable. The main purpose of this paper is to improve the allowable upper bounds of impulse. In our new stability criterion, the harsh condition (1) is unnecessary.

This paper is organized as follows. In Section 2, the new BAM neural network model is formulated, and some necessary preparation knowledge is provided. In Section 3, we firstly employ variational methods to obtain an inequalities lemma and then use the Lyapunov functional method and Schur Complement technique to deduce a LMI-based exponential stability criterion. In Section 4 , an example is provided to illustrate the effectiveness of the proposed methods. In the end, Section 5 contains some conclusions of this paper.

Remark 1. In [24, 25], a class of delay differential inequalities ([24, Lemma 2.2] and [25, Lemma 3]) were employed to obtain the stability criteria for deterministic systems. However, a stability criterion of Markovian jumping stochastic system can be obtained via Gronwall-Bellman-type impulsive integral inequality in this paper. To some extent, the restrictive conditions of Gronwall-Bellman-type impulsive integral inequality lemma are simpler than those of the delay differential inequality lemmas ([24, Lemma 2.2] and [25, Lemma 3]), for there are some advantages of the utilization of Gronwall-Bellman-type inequalities (e.g., the allowable upper bounds of time-delays), which will be illustrated in Numerical Example.

\section{Model Description and Preliminaries}

In 1987, the bidirectional associative memory (BAM) neural networks were introduced by Kosko (see $[1,26])$. He set up the following mathematical model [1]:

$$
\begin{aligned}
& \dot{x}_{i}=-a_{i} x_{i}(t)+\sum_{j=1}^{p} w_{j i} g_{j}\left(y_{j}(t)\right)+I_{i}, \quad i=1,2, \ldots, n, \\
& \dot{y}_{j}=-b_{j} y_{j}(t)+\sum_{i=1}^{n} v_{i j} g_{i}\left(x_{i}(t)\right)+J_{j}, \quad j=1,2, \ldots, n .
\end{aligned}
$$

We can know from the above model that it has generalized the single-layer autoassociative Hebbian correlator to twolayer pattern-matched heteroassociative circuits. Neurons are placed in the two layers, and neurons of the same layer are not connected while the neurons of the different layers are connected. There exists the bidirectional information transfer between the two layers of neurons. Such class of networks has wide applications in many fields such as pattern recognition, associative memory, and artificial intelligence. But the important precondition of these applications is that the system should be stable. So Kosko proved the stability of the above model in [1]. Since then, the stability analysis of the BAM neural network becomes the most active area of research ([26 ] and their references therein). For a power system of signal transmission, there inevitably is a time lag problem. And the existence of time-delay often results in unstable phenomenon of a network system. Besides, in the real world, impulsive phenomena exist in the process of changing dynamic behaviors. In order to give an exact description of these process, adoption of delay impulsive differential equations for BAM neural networks is a more effective method (see [3, 4, 27-29] and their references therein). In addition, the BAM neural networks are often disturbed by environmental noise. The noise may influence the stability of the equilibrium and vary some structure parameters, which usually satisfies the Markov process $([30,31]$ and their references therein). And diffusion effect exists really in the BAM neural networks when electrons are moving in asymmetric electromagnetic fields ([2-6] and their references therein).

Often we assume that there exits an equilibrium point for BAM neural networks. By using the translation transform regarding the equilibrium point, we can actually consider the following BAM neural networks which owns the null solution as its equilibrium point:

$$
\begin{aligned}
& \frac{\partial u}{\partial t}= \nabla(D(t, x, u) \circ \nabla u)-A(r(t)) u(t, x) \\
&+W_{1}(r(t)) f_{1}(v(t, x)) \\
&+W_{2}(r(t)) f_{2}(v(t-\tau(t), x)), \\
& x \in \Omega, t \neq t_{k}, \\
& \frac{\partial v}{\partial t}= \cdot(\mathscr{D}(t, x, v) \circ \nabla v)-B(r(t)) v(t, x) \\
&+\Gamma_{1}(r(t)) g_{1}(u(t, x)) \\
&+\Gamma_{2}(r(t)) g_{2}(u(t-\tau(t), x)), \quad x \in \Omega, t \neq t_{k}, \\
& \Delta u\left(t_{k}, x\right)=\left(C_{k}(r(t))-I\right) u\left(t_{k}, x\right), \Delta v\left(t_{k}, x\right) \\
&=\left(\mathscr{C}_{k}(r(t))-I\right) v\left(t_{k}, x\right),
\end{aligned}
$$

equipped with the initial condition

$$
\begin{aligned}
& u\left(s, x ; t_{0}, \varphi\right)=\varphi(s, x), \\
& v\left(s, x ; t_{0}, \psi\right)=\psi(s, x), \\
& t_{0}-\tau \leqslant s \leqslant t_{0}, x \in \Omega,
\end{aligned}
$$

and the Dirichlet boundary value condition

$$
\begin{aligned}
& u\left(s, x ; t_{0}, \varphi\right)=0, \\
& v\left(s, x ; t_{0}, \varphi\right)=0,
\end{aligned}
$$


where $x \in \Omega, \Omega$ is a bounded domain in $R^{m}$ with a smooth boundary $\partial \Omega$ of class $\mathscr{C}^{2}$ by $\Omega$ (see, e.g., [32]), $u=u(t$, $x)=\left(u_{1}(t, x), u_{2}(t, x), \ldots, u_{n}(t, x)\right)^{T}, v=v(t, x)=\left(v_{1}(t, x)\right.$, $\left.v_{2}(t, x), \ldots, v_{n}(t, x)\right)^{T} \in R^{n}$, and $u_{i}(t, x)$ and $v_{j}(t, x)$ are state variables of the $i$ th neuron and the $j$ th neuron at time $t$ and in space variable $x . f_{i}(v)=f_{i}(v(t, x))=\left(f_{1}^{(i)}\left(v_{1}(t\right.\right.$, $\left.x)), \ldots, f_{n}^{(i)}\left(v_{n}(t, x)\right)\right)^{T}, g_{i}(u)=g_{i}(u(t, x))=\left(g_{1}^{(i)}\left(u_{1}(t, x)\right)\right.$, $\left.\ldots, g_{n}^{(i)}\left(u_{n}(t, x)\right)\right)^{T}, i=1,2$, and $f_{j}^{(i)}\left(u_{j}(t, x)\right)$ and $g_{j}\left(u_{j}^{(i)}(t\right.$, $x)$ ) are neuron activation functions of the $j$ th unit at time $t$ and in space variable $x . \Delta u\left(t_{k}, x\right)=u\left(t_{k}^{+}, x\right)-u\left(t_{k}, x\right)$ with $u\left(t_{k}^{-}, x\right)=u\left(t_{k}, x\right)$ and $\Delta v\left(t_{k}, x\right)=v\left(t_{k}^{+}, x\right)-v\left(t_{k}, x\right)$ with $v\left(t_{k}^{-}, x\right)=v\left(t_{k}, x\right) .(D(t, x, u) \circ \nabla u)$ is Hadamard product of matrices $(D(t, x, u))_{n \times m}$ and $\nabla u$. The definition of Hadamard product may be seen in $[7] .(\widehat{\Omega}, \Upsilon, \mathbb{P})$ is the given probability space where $\widehat{\Omega}$ is sample space, $\Upsilon$ is $\sigma$-algebra of subset of the sample space, and $\mathbb{P}$ is the probability measure defined on $\Upsilon$. Let $S=\{1,2, \ldots, N\}$ and the random form process $\{r(t), t \in[0,+\infty)\}$ be a homogeneous, finite-state Markovian process with right continuous trajectories with generator $\Pi=$ $\left(\pi_{i j}\right)_{N \times N}$ and transition probability from mode $i$ at time $t$ to mode $j$ at time $t+\Delta t, i, j \in S$,

$$
\begin{aligned}
\mathbb{P} & (r(t+\delta)=j \mid r(t)=i) \\
& = \begin{cases}\pi_{i j} \delta+o(\delta), & j \neq i \\
1+\pi_{i j} \delta+o(\delta), & j=i,\end{cases}
\end{aligned}
$$

where $\pi_{i j} \geqslant 0$ is transition probability rate from $i$ to $j(j \neq i)$ and $\pi_{i i}=-\sum_{j=1, j \neq i}^{s} \pi_{i j}, \delta>0$, and $\lim _{\delta \rightarrow 0} o(\delta) / \delta=0$. For any given $r(t)=r \in S$, we denote $A_{r}=A(r(t)), B_{r}=B(r(t))$, $W_{i r}=W_{i}(r(t))$, and $\Gamma_{i r}=\Gamma_{i}(r(t))$ for $i=1,2$. Define the diagonal matrices $C_{k r}=C_{k}(r(t))$ and $\mathscr{C}_{k r}=\mathscr{C}_{k}(r(t))$. That is, $C_{k r}=\operatorname{diag}\left(c_{k r}^{(1)}, c_{k r}^{(2)}, \ldots, c_{k r}^{(n)}\right)$ and $\mathscr{C}_{k r}=\operatorname{diag}\left(\bar{c}_{k r}^{(1)}\right.$, $\left.\bar{c}_{k r}^{(2)}, \ldots, \bar{c}_{k r}^{(n)}\right)$. Also, $A_{r}$ and $B_{r}$ both are diagonal matrices. Time-delays $0 \leqslant \tau(t) \leqslant \tau$ and $\dot{\tau}(t)<1-1 / h$ with $h>0$.

There exist positive definite diagonal matrices $H_{i}$ and $\mathscr{H}_{i}$ such that

$$
\begin{aligned}
& \left|f_{i}(u)-f_{i}(v)\right| \leqslant H_{i}|u-v| ; \\
& \left|g_{i}(u)-g_{i}(v)\right| \leqslant \mathscr{H}_{i}|u-v|,
\end{aligned}
$$

for $u, v \in R^{n}, i=1,2$,

where we denote $|u|=\left(\left|u_{1}\right|,\left|u_{2}\right|, \ldots,\left|u_{n}\right|\right)^{T},\left|f_{i}(u)\right|=$ $\left(\left|f_{1}^{(i)}\left(u_{1}\right)\right|,\left|f_{2}^{(i)}\left(u_{2}\right)\right|, \ldots,\left|f_{n}^{(i)}\left(u_{n}\right)\right|\right)^{T}$, and $\left|g_{i}(u)\right|=\left(\left|g_{1}^{(i)}\left(u_{1}\right)\right|\right.$, $\left.\left|g_{2}^{(i)}\left(u_{2}\right)\right|, \ldots,\left|g_{n}^{(i)}\left(u_{n}\right)\right|\right)^{T}$.

Throughout this paper, we assume that $f_{i}(0)=g_{i}(0)=0$ for $i=1,2$. Then $u=0$ and $v=0$ is the null solution for problem (3)-(5).

Definition 2. For symmetric matrices $A$ and $B$, one denotes $A<B$ or $B>A$ if matrix $B-A$ is a positive definite matrix. Particularly, $A>0$ if symmetric matrix $A$ is a positive definite matrix.

Let $u\left(t, x ; \varphi, \psi, i_{0}\right)$ and $v\left(t, x ; \varphi, \psi, i_{0}\right)$ denote the state trajectory from the initial condition $r\left(t_{0}\right)=i_{0}$, $u\left(t_{0}+\theta, x ; \varphi, \psi\right)=\varphi(\theta, x)$, and $v\left(t_{0}+\theta, x ; \varphi, \psi\right)=\psi(\theta, x)$ on $t_{0}-\tau \leqslant \theta \leqslant t_{0}$ in $L_{\mathscr{F}_{0}}^{2}\left([-\tau, 0] \times \Omega ; R^{n}\right)$. Here, $L_{\mathscr{F}_{0}}^{2}([-\tau$, $\left.0] \times \Omega ; R^{n}\right)$ denotes the family of all $\mathscr{F}_{0}$-measurable $\mathscr{C}\left([-\tau, 0] \times \Omega ; R^{n}\right)$-value random variable $\xi=\{\xi(\theta, x)$ : $\left.t_{0}-\tau \leqslant \theta \leqslant t_{0}, \quad x \in \Omega\right\}$ such that $\sup _{t_{0}-\tau \leqslant \theta \leqslant t_{0}} \mathbb{E}\|\xi(\theta)\|^{2}<\infty$, where $\mathbb{E}\{\cdot\}$ stands for the mathematical expectation operator with respect to the given probability measure $\mathbb{P}$.

Definition 3. The equilibrium point $u=0$ and $v=0$ of problem (3)-(5) is said to be globally stochastically exponential stability if, for every initial condition $\varphi, \psi \in L_{\mathscr{F}_{0}}^{2}([-\tau, 0] \times$ $\left.\Omega ; R^{n}\right), r\left(t_{0}\right)=i_{0}$, there exist scalars $c_{1}>0, c_{2}>0, \beta>0$, and $\gamma>0$ such that, for any solution $u\left(t, x ; \varphi, \psi, i_{0}\right), v(t$, $\left.x ; \varphi, \psi, i_{0}\right)$,

$$
\begin{aligned}
& \mathbb{E}\left(\left\|u\left(t, x ; \varphi, \psi, i_{0}\right)\right\|^{2}\right)+\mathbb{E}\left(\left\|v\left(t, x ; \varphi, \psi, i_{0}\right)\right\|^{2}\right) \\
& \quad \leqslant \gamma e^{-\beta\left(t-t_{0}\right)}\left[c_{1} \mathbb{E}\left(\|\varphi\|_{\tau}^{2}\right)+c_{2} \mathbb{E}\left(\|\psi\|_{\tau}^{2}\right)\right], \quad t \geqslant t_{0},
\end{aligned}
$$

where

$$
\begin{aligned}
& \left\|u\left(t, x ; \varphi, \psi, i_{0}\right)\right\|^{2} \\
& \quad=\int_{\Omega} u^{T}\left(t, x ; \varphi, \psi, i_{0}\right) u\left(t, x ; \varphi, \psi, i_{0}\right) d x, \\
& \left\|v\left(t, x ; \varphi, \psi, i_{0}\right)\right\|^{2} \\
& \quad=\int_{\Omega} v^{T}\left(t, x ; \varphi, \psi, i_{0}\right) v\left(t, x ; \varphi, \psi, i_{0}\right) d x, \\
& \|\varphi\|_{\tau}^{2}=\sup _{t_{0}-\tau \leqslant s \leqslant t_{0}} \int_{\Omega} \varphi^{T}(s, x) \varphi(s, x) d x, \\
& \|\psi\|_{\tau}^{2}=\sup _{t_{0}-\tau \leqslant s \leqslant t_{0}} \int_{\Omega} \psi^{T}(s, x) \psi(s, x) d x .
\end{aligned}
$$

Throughout this paper, we define $\lambda_{1}=\min \left\{\int_{\Omega}|\nabla u|^{2} d x\right.$ : $\left.u \in W_{0}^{1,2}(\Omega), \int_{\Omega}|u|^{2} d x=1\right\}$, the first eigenvalue of $-\Delta$ in Sobolev space $W_{0}^{1,2}(\Omega)[33]$.

Lemma 4 ((see [8]) (Gronwall-Bellman-type impulsive integral inequality)). Assume that

(1) the sequence $\left\{t_{k}\right\}$ satisfies $0 \leqslant t_{0}<t_{1}<t_{2}<\cdots$ with $\lim _{k \rightarrow \infty} t_{k}=\infty$,

(2) $q \in P C^{1}\left[R^{+}, R\right]$ and $q(t)$ is left-continuous at $t_{k}, k=$ $1,2, \ldots$,

(3) $p \in C\left[R^{+}, R^{+}\right]$and for $k=1,2, \ldots$,

$$
q(t) \leqslant c+\int_{t_{0}}^{t} p(s) q(s) d s+\sum_{t_{0}<t_{k}<t} \eta_{k} q\left(t_{k}\right), \quad t \geqslant t_{0},
$$

where $\eta_{k} \geqslant 0$ and $c=$ const.

Then

$$
q(t) \leqslant c \prod_{t_{0}<t_{k}<t}\left(1+\eta_{k}\right) e^{\int_{t_{0}}^{t} p(s) d s}, \quad t \geqslant t_{0} .
$$


Lemma 5 (see [34]). Let $x \in R^{n}, y \in R^{n}$, and $\varepsilon>0$. Then one has

$$
x^{T} y+y^{T} x \leqslant \varepsilon x^{T} x+\varepsilon^{-1} y^{T} y .
$$

Lemma 6 (Schur Complement [35]). Given matrices $Q(t)$, $\mathcal{S}(t)$, and $\mathscr{R}(t)$ with appropriate dimensions, where $\mathbb{Q}(t)=$ $\mathbb{Q}(t)^{T}, \mathscr{R}(t)=\mathscr{R}(t)^{T}$, then

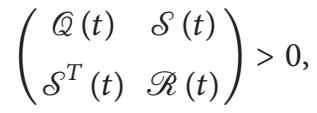

if and only if

$$
\mathscr{R}(t)>0, \quad \mathbb{Q}(t)-\mathcal{S}(t) \mathscr{R}^{-1}(t) \mathcal{S}^{T}(t)>0
$$

or

$$
\mathbb{Q}(t)>0, \quad \mathscr{R}(t)-\mathcal{S}^{T}(t) \mathscr{Q}^{-1}(t) \mathcal{S}^{T}(t)>0,
$$

where $Q(t), \mathcal{S}(t)$, and $\mathscr{R}(t)$ are dependent on $t$.

\section{Main Results}

Throughout this paper, we define $\lambda_{1}=\min \left\{\int_{\Omega}|\nabla u|^{2} d x: u \in\right.$ $\left.H_{0}^{1}(\Omega), \int_{\Omega}|u|^{2} d x=1\right\}$, the first eigenvalue of $-\Delta$ in Sobolev space $H_{0}^{1}(\Omega)[18,33]$.

Lemma 7. Let $P_{r}=\operatorname{diag}\left(p_{r 1}, p_{r 2}, \ldots, p_{r n}\right)$ be a positive definite matrix, $\underline{\alpha}>0$ with $\underline{\alpha} I \leqslant P_{r}$, and let $u$ and $v$ be a solution of problem (3)-(5). Then one has

$$
\begin{aligned}
& \int_{\Omega} u^{T} P_{r} \nabla \cdot(D \circ \nabla u) d x \leqslant-\lambda_{1} \underline{\alpha} d\|u\|^{2}, \\
& \int_{\Omega} v^{T} P_{r} \nabla \cdot(\mathscr{D} \circ \nabla v) d x \leqslant-\lambda_{1} \underline{\alpha} \widetilde{d}\|\|^{2},
\end{aligned}
$$

where $d=\min _{i, j}\left\{\inf _{\left[t_{0},+\infty\right) \times \Omega \times R} D_{i j}(t, x, u)\right\}$ and $\widetilde{d}=$ $\min _{i, j}\left\{\inf _{\left[t_{0},+\infty\right) \times \Omega \times R} \mathscr{D}_{i j}(t, x, v)\right\}$.

Proof. Since $u$ and $v$ is a solution of problem (3)-(5), it follows by Gauss formula and the Dirichlet boundary condition that

$$
\begin{aligned}
& \int_{\Omega} u^{T} P_{r}(\nabla \cdot(D \circ \nabla u)) d x \\
& \quad=\int_{\Omega} u^{T} P_{r}\left(\sum_{k=1}^{m} \frac{\partial}{\partial x_{k}}\left(D_{1 k} \frac{\partial u_{1}}{\partial x_{k}}\right), \ldots,\right. \\
& \left.\sum_{k=1}^{m} \frac{\partial}{\partial x_{k}}\left(D_{n k} \frac{\partial u_{n}}{\partial x_{k}}\right)\right)^{T} d x \\
& =-\sum_{k=1}^{m} \sum_{j=1}^{n} \int_{\Omega} p_{r j} D_{j k}\left(\frac{\partial u_{j}}{\partial x_{k}}\right)^{2} d x \leqslant-\lambda_{1} \underline{\alpha} d\|u\|^{2} .
\end{aligned}
$$

Similarly, we can prove that $\int_{\Omega} v^{T} P_{r} \nabla \cdot(\mathscr{D} \circ \nabla v) d x \leqslant$ $-\lambda_{1} \underline{\alpha} \widetilde{d}\|v\|^{2}$. Thus, the proof is completed.
Theorem 8. Assume that there are positive scalars $\mu$ and $\alpha$, satisfying $\inf _{k=1,2, . .}\left(t_{k}-t_{k-1}\right) \geqslant \mu$ and

$$
\begin{aligned}
& -\sqrt{1+\alpha} I \leqslant C_{k r} \leqslant \sqrt{1+\alpha} I, \\
& -\sqrt{1+\alpha} I \leqslant \mathscr{C}_{k r} \leqslant \sqrt{1+\alpha} I,
\end{aligned}
$$

$$
r \in S, k=1,2, \ldots
$$

Assume, in addition, that there exist positive definite matrices $P_{r}=\operatorname{diag}\left(p_{r 1}, p_{r 2}, \ldots, p_{r n}\right), r \in S$, and positive scalars $\underline{\alpha}, \bar{\alpha}, \beta_{1}$, and $\beta_{2}$ such that the following LMIs conditions are satisfied:

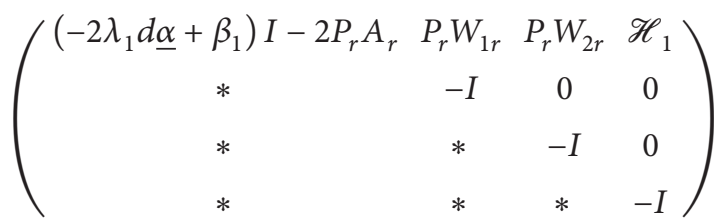

$<0, \quad \forall r \in S$,

$$
\begin{gathered}
<0, \quad \forall r \in S, \\
\underline{\alpha} I \leqslant P_{r}, \quad \forall r \in S, \\
P_{r} \leqslant \bar{\alpha} I, \quad \forall r \in S, \\
\left(\begin{array}{cr}
-\beta_{2} I & H_{2} \\
* & -I
\end{array}\right)<0,
\end{gathered}
$$$$
\left(\begin{array}{cccc}
\left(-2 \lambda_{1} \tilde{d} \underline{\alpha}+\beta_{1}\right) I-2 P_{r} B_{r} & P_{r} \Gamma_{1 r} & P_{r} \Gamma_{2 r} & H_{1} \\
* & -I & 0 & 0 \\
* & * & -I & 0 \\
* & * & * & -I
\end{array}\right)
$$

$\left(\begin{array}{cc}-\beta_{2} I & \mathscr{H}_{2} \\ * & -I\end{array}\right)<0$

where $I$ is the identity matrix, $d=\min _{i, j}\left\{\inf _{\left[t_{0},+\infty\right) \times \Omega \times R} D_{i j}(t\right.$, $x, u)\}, \widetilde{d}=\min _{i, j}\left\{\inf _{\left[t_{0},+\infty\right) \times \Omega \times R} \mathscr{D}_{i j}(t, x, v)\right\}$, and

$$
\frac{\beta_{1}}{\bar{\alpha}}-\frac{\ln (1+\alpha)}{\mu}-\sum_{j \in S}^{j \neq r} \frac{\pi_{r j} \bar{\alpha}}{\underline{\alpha}}-\frac{\beta_{2} h e^{\gamma \tau}}{\underline{\alpha}}>0, \quad \forall r \in S .
$$

Then the equilibrium point $u=0$ and $v=0$ of problem (3)(5) is globally stochastically exponential stability.

Proof. Consider the following Lyapunov-Krasovskii functional:

$$
\begin{aligned}
& V(t, r)=e^{\gamma\left(t-t_{0}\right)} \int_{\Omega}\left(u^{T}(t, x) P(r(t)) u(t, x)\right. \\
& \left.\quad+v^{T}(t, x) P(r(t)) v(t, x)\right) d x, \quad \forall r(t)=r \in S .
\end{aligned}
$$


Let $L$ be the weak infinitesimal operator such that

$$
\begin{aligned}
& L V(t, r)=e^{\gamma\left(t-t_{0}\right)} \int_{\Omega}\left[u^{T}\left(\gamma P_{r}+\sum_{j \in S} \pi_{r j} P_{j}\right) u\right. \\
& \left.+v^{T}\left(\gamma P_{r}+\sum_{j \in S} \pi_{r j} P_{j}\right) v\right] d x \\
& +e^{\gamma\left(t-t_{0}\right)} \int_{\Omega}\left[-2 \sum_{k=1}^{m} \sum_{j=1}^{n} p_{r j} D_{j k}\left(\frac{\partial u_{j}}{\partial x_{k}}\right)^{2}\right. \\
& +2 u^{T} P_{r} A_{r} u+f_{1}^{T}(v) W_{1 r}^{T} P_{r} u+u^{T} P_{r} W_{1 r} f_{1}(v) \\
& +f_{2}^{T}(v(t-\tau(t), x)) W_{2 r}^{T} P_{r} u \\
& +u^{T} P_{r} W_{2 r} f_{2}(v(t-\tau(t), x)) \\
& +2 \sum_{k=1}^{m} \sum_{j=1}^{n} p_{r j} \mathscr{D}_{j k}\left(\frac{\partial v_{j}}{\partial x_{k}}\right)^{2}-2 v^{T} P_{r} B_{r} v \\
& +g_{1}^{T}(u) \Gamma_{1 r}^{T} P_{r} v+v^{T} P_{r} \Gamma_{1 r} g_{1}(u) \\
& +g_{2}^{T}(u(t-\tau(t), x)) \Gamma_{2 r}^{T} P_{r} v \\
& \left.+v^{T} P_{r} \Gamma_{2 r} g_{2}(u(t-\tau(t), x))\right] d x .
\end{aligned}
$$

In view of

$$
\int_{\Omega} u^{T} P_{j} u d x \leqslant \bar{\alpha} \int_{\Omega} u^{T} I u d x \leqslant \bar{\alpha} \int_{\Omega} u^{T} \frac{1}{\underline{\alpha}} P_{r} u d x,
$$

we have

$$
\begin{aligned}
& L V(t, r) \leqslant\left(\gamma+\sum_{j \in S}^{j \neq r} \pi_{r j} \frac{\bar{\alpha}}{\alpha}\right) V(t, r) \\
& +e^{\gamma\left(t-t_{0}\right)} \int_{\Omega}\left[-2 \sum_{k=1}^{m} \sum_{j=1}^{n} p_{r j} D_{j k}\left(\frac{\partial u_{j}}{\partial x_{k}}\right)^{2}\right. \\
& +2 u^{T} P_{r} A_{r} u+f_{1}^{T}(v) W_{1 r}^{T} P_{r} u+u^{T} P_{r} W_{1 r} f_{1}(v) \\
& +f_{2}^{T}(v(t-\tau(t), x)) W_{2 r}^{T} P_{r} u \\
& +u^{T} P_{r} W_{2 r} f_{2}(v(t-\tau(t), x)) \\
& +2 \sum_{k=1}^{m} \sum_{j=1}^{n} p_{r j} \mathscr{D}_{j k}\left(\frac{\partial v_{j}}{\partial x_{k}}\right)^{2}-2 v^{T} P_{r} B_{r} v \\
& +g_{1}^{T}(u) \Gamma_{1 r}^{T} P_{r} v+v^{T} P_{r} \Gamma_{1 r} g_{1}(u) \\
& +g_{2}^{T}(u(t-\tau(t), x)) \Gamma_{2 r}^{T} P_{r} v \\
& \left.+v^{T} P_{r} \Gamma_{2 r} g_{2}(u(t-\tau(t), x))\right] d x .
\end{aligned}
$$

It follows by the Lipschitz assumption (7) and Lemma 5 that

$$
\begin{aligned}
& \int_{\Omega}\left[f_{2}^{T}(v(t-\tau(t), x)) W_{2 r}^{T} P_{r} u\right. \\
& \left.\quad+u^{T} P_{r} W_{2 r} f_{2}(v(t-\tau(t), x))\right] d x \\
& \quad \leqslant \int_{\Omega}\left[f_{2}^{T}(v(t-\tau(t), x)) f_{2}(v(t-\tau(t), x))\right. \\
& \left.\quad+\left(W_{2 r}^{T} P_{r} u\right)^{T}\left(W_{2 r}^{T} P_{r} u\right)\right] d x \\
& \quad \leqslant \int_{\Omega}\left[v^{T}(t-\tau(t), x) H_{2}^{2} v(t-\tau(t), x)\right. \\
& \left.\quad+u^{T} P_{r}\left(W_{2 r} W_{2 r}^{T}\right) P_{r} u\right] d x .
\end{aligned}
$$

Similarly, we have

$$
\begin{aligned}
& \int_{\Omega}\left[f_{1}^{T}(v(t, x)) W_{1 r}^{T} P_{r} u+u^{T} P_{r} W_{1 r} f_{1}(v(t, x))\right] d x \\
& \leqslant \int_{\Omega}\left[v^{T} H_{1}^{2} v+u^{T}\left(P_{r} W_{1 r} W_{1 r}^{T} P_{r}\right) u\right] d x, \\
& \int_{\Omega}\left[g_{1}^{T}(u(t, x)) \Gamma_{1 r}^{T} P_{r} v+v^{T} P_{r} \Gamma_{1 r} g_{1}(u(t, x))\right] d x \\
& \leqslant \int_{\Omega}\left[u^{T} \mathscr{H}_{1}^{2} u+v^{T}\left(P_{r} \Gamma_{1 r} \Gamma_{1 r}^{T} P_{r}\right) v\right] d x, \\
& \int_{\Omega}\left[g_{2}^{T}(u(t-\tau(t), x)) \Gamma_{2 r}^{T} v\right. \\
& \left.\quad+v^{T} \Gamma_{2 r} g_{2}(u(t-\tau(t), x))\right] d x \\
& \quad \leqslant \int_{\Omega}\left[u^{T}(t-\tau(t), x) \mathscr{H}_{2}^{2} u(t-\tau(t), x)\right. \\
& \left.\quad+v^{T}\left(P_{r} \Gamma_{2 r} \Gamma_{2 r}^{T} P_{r}\right) v\right] d x .
\end{aligned}
$$

From the above analysis, Lemma 7, (19), (20), (23), (24), and Schur Complement theorem, we can deduce

$$
\begin{aligned}
& L V(t, r) \leqslant\left(\gamma+\sum_{j \in S}^{j \neq r} \pi_{r j} \frac{\bar{\alpha}}{\alpha}\right) V(t, r) \\
& -e^{\gamma\left(t-t_{0}\right)} \int_{\Omega}\left[u ^ { T } \left(2 \lambda_{1} d \underline{\alpha} I+2 P_{r} A_{r}\right.\right. \\
& \left.-P_{r}\left(W_{1 r} W_{1 r}^{T}+W_{2 r} W_{2 r}^{T}\right) P_{r}-\mathscr{H}_{1}^{2}\right) u \\
& +v^{T}\left(2 \lambda_{1} \tilde{d} \underline{\alpha} I+2 P_{r} B_{r}-P_{r}\left(\Gamma_{1 r} \Gamma_{1 r}^{T}+\Gamma_{2 r} \Gamma_{2 r}^{T}\right) P_{r}\right. \\
& \left.\left.-H_{1}^{2}\right) v\right] d x+e^{\gamma\left(t-t_{0}\right)} \int_{\Omega}\left[u^{T}(t-\tau(t), x)\right. \\
& \cdot \mathscr{H}_{2}^{2} u(t-\tau(t), x)+v^{T}(t-\tau(t), x) H_{2}^{2} v(t \\
& -\tau(t), x)] d x \leqslant\left(\gamma+\sum_{j \in S}^{j \neq r} \pi_{r j} \frac{\bar{\alpha}}{\alpha}\right) V(t, r)
\end{aligned}
$$




$$
\begin{aligned}
& -e^{\gamma\left(t-t_{0}\right)} \int_{\Omega}\left[\beta_{1}\left(u^{T} u+v^{T} v\right)\right] d x \\
& +e^{\gamma\left(t-t_{0}\right)} \int_{\Omega}\left[\beta _ { 2 } \left(u^{T}(t-\tau(t), x) u(t-\tau(t), x)\right.\right. \\
& \left.\left.+v^{T}(t-\tau(t), x) v(t-\tau(t), x)\right)\right] d x \leqslant(\gamma \\
& \left.+\sum_{j \in S}^{j \neq r} \pi_{r j} \frac{\bar{\alpha}}{\alpha}\right) V(t, r)-\frac{\beta_{1}}{\bar{\alpha}} V(t, r)+\beta_{2} \widetilde{V}(t \\
& -\tau(t), r),
\end{aligned}
$$

where $\widetilde{V}(t-\tau(t), r)=e^{\gamma\left(t-t_{0}\right)} \int_{\Omega}\left[\left(u^{T}(t-\tau(t), x) u(t-\tau(t), x)+\right.\right.$ $\left.\left.v^{T}(t-\tau(t), x) v(t-\tau(t), x)\right)\right] d x$.

Then we can derive that, for $t \in\left(t_{k}, t_{k+1}\right)$,

$$
\begin{aligned}
L V(t, r) \leqslant & \left(\gamma+\sum_{j \in S}^{j \neq r} \frac{\pi_{r j} \bar{\alpha}}{\alpha}-\frac{\beta_{1}}{\bar{\alpha}}\right) V(t, r) \\
& +\beta_{2} \widetilde{V}(t-\tau(t), r) \\
\leqslant & \xi V(t, r)+\beta_{2} \widetilde{V}(t-\tau(t), r),
\end{aligned}
$$

where $\xi=\gamma-\beta_{1} / \bar{\alpha}+\max _{r \in S}\left(\sum_{j \in S}^{j \neq r}\left(\pi_{r j} \bar{\alpha} / \underline{\alpha}\right)\right)$.

Let $\varepsilon>0$ small enough, and we can integrate (33) from $t_{k}+\varepsilon$ to $t$ as follows:

$$
\begin{gathered}
\mathbb{E} V(t, r) \leqslant \mathbb{E} V\left(t_{k}+\varepsilon, r\right)+\xi \int_{t_{k}+\varepsilon}^{t} \mathbb{E} V(s, r) d s \\
+\beta_{2} \int_{t_{k}+\varepsilon}^{t} \mathbb{E} \widetilde{V}(s-\tau(s), r) d s .
\end{gathered}
$$

Let $\varepsilon \rightarrow 0$, and then we have

$$
\begin{aligned}
\mathbb{E} V(t, r) \leqslant & \mathbb{E} V\left(t_{k}^{+}, r\right)+\xi \int_{t_{k}}^{t} \mathbb{E} V(s, r) d s \\
& +\beta_{2} \int_{t_{k}}^{t} \mathbb{E} \widetilde{V}(s-\tau(s), r) d s, \\
& \quad t \in\left(t_{k}, t_{k+1}\right), k=0,1,2, \ldots
\end{aligned}
$$

Let $t=t_{k+1}-\varepsilon$ with $\varepsilon>0$ small enough, and we can get by (35)

$$
\begin{aligned}
\mathbb{E} V\left(t_{k+1}-\varepsilon, r\right) \leqslant & \mathbb{E} V\left(t_{k}^{+}, r\right)+\xi \int_{t_{k}}^{t_{k+1}-\varepsilon} \mathbb{E} V(s, r) d s \\
& +\beta_{2} \int_{t_{k}}^{t_{k+1}-\varepsilon} \mathbb{E} \widetilde{V}(s-\tau(s), r) d s .
\end{aligned}
$$

Moreover, let $\varepsilon \rightarrow 0$, and then we can get by $\mathbb{E} V\left(t_{k+1}^{-}\right)=$ $\mathbb{E} V\left(t_{k+1}\right)$

$$
\begin{aligned}
\mathbb{E} V\left(t_{k+1}, r\right) \leqslant & \mathbb{E} V\left(t_{k}^{+}, r\right)+\xi \int_{t_{k}}^{t} \mathbb{E} V(s, r) d s \\
& +\beta_{2} \int_{t_{k}}^{t} \mathbb{E} \widetilde{V}(s-\tau(s), r) d s .
\end{aligned}
$$

Combining (35) and (37) results in

$$
\begin{aligned}
& \mathbb{E} V(t, r) \leqslant \mathbb{E} V\left(t_{k}^{+}, r\right)+\xi \int_{t_{k}}^{t} \mathbb{E} V(s, r) d s \\
&+\beta_{2} \int_{t_{k}}^{t} \mathbb{E} \widetilde{V}(s-\tau(s), r) d s, \\
& \quad t \in\left(t_{k}, t_{k+1}\right], k=0,1,2, \ldots
\end{aligned}
$$

On the other hand, since both $C_{k r}$ and $\mathscr{C}_{k r}$ are diagonal matrices, we can get by (18)

$$
\begin{aligned}
u^{T}\left(t_{k}^{+}, x\right) P_{r} u\left(t_{k}^{+}, x\right) & =u^{T}\left(t_{k}, x\right) C_{k r} P_{r} C_{k r} u\left(t_{k}, x\right) \\
& =u^{T}\left(t_{k}, x\right) P_{r} C_{k r}^{2} u\left(t_{k}, x\right) \\
& \leqslant(1+\alpha) u^{T}\left(t_{k}, x\right) P_{r} u\left(t_{k}, x\right), \\
v^{T}\left(t_{k}^{+}, x\right) P_{r} v\left(t_{k}^{+}, x\right) & =v^{T}\left(t_{k}, x\right) \mathscr{C}_{k r} P_{r} \mathscr{C}_{k r} v\left(t_{k}, x\right) \\
& \leqslant(1+\alpha) v^{T}\left(t_{k}, x\right) P_{r} v\left(t_{k}, x\right) .
\end{aligned}
$$

Hence,

$$
\mathbb{E} V\left(t_{k}^{+}, r\right) \leqslant(1+\alpha) \mathbb{E} V\left(t_{k}, r\right) .
$$

Synthesizing (38) and (40) results in

$$
\begin{aligned}
\mathbb{E} V(t, r) \leqslant & \mathbb{E} V\left(t_{k}, r\right)+\alpha \mathbb{E} V\left(t_{k}, r\right) \\
& +\xi \int_{t_{k}}^{t} \mathbb{E} V(s, r) d s \\
& +\beta_{2} \int_{t_{k}}^{t} \mathbb{E} \widetilde{V}(s-\tau(s), r) d s, \\
& \quad t \in\left(t_{k}, t_{k+1}\right], k=0,1,2, \ldots
\end{aligned}
$$

By induction argument, we have

$$
\begin{gathered}
\mathbb{E} V\left(t_{k}, r\right) \leqslant \mathbb{E} V\left(t_{k-1}, r\right)+\alpha \mathbb{E} V\left(t_{k-1}, r\right) \\
+\xi \int_{t_{k-1}}^{t_{k}} \mathbb{E} V(s, r) d s+\beta_{2} \int_{t_{k-1}}^{t_{k}} \mathbb{E} \widetilde{V}(s-\tau(s), r) d s \\
\vdots \\
\mathbb{E} V\left(t_{1}, r\right) \leqslant \mathbb{E} V\left(t_{0}, r\right)+\alpha \mathbb{E} V\left(t_{0}, r\right) \\
+\xi \int_{t_{0}}^{t_{1}} \mathbb{E} V(s, r) d s+\beta_{2} \int_{t_{0}}^{t_{1}} \mathbb{E} \widetilde{V}(s-\tau(s), r) d s .
\end{gathered}
$$


Hence, for $k=0,1,2, \ldots$,

$$
\mathbb{E} V(t, r) \leqslant \mathbb{E} V\left(t_{0}, r\right)+\alpha \mathbb{E} V\left(t_{0}, r\right)
$$$$
+\alpha \sum_{t_{0}<t_{k}<t} \mathbb{E} V\left(t_{k}, r\right)+\xi \int_{t_{0}}^{t} \mathbb{E} V(s, r) d s
$$

$$
+\beta_{2} \int_{t_{0}}^{t} \mathbb{E} \widetilde{V}(s-\tau(s), r) d s,
$$

$t \in\left(t_{k}, t_{k+1}\right]$.

Since time-delays $0 \leqslant \tau(t) \leqslant \tau$ and $\dot{\tau}(t)<1-1 / h$ with $h>0$, we have

$$
\begin{aligned}
& \int_{t_{0}}^{t} \mathbb{E} \widetilde{V}(s-\tau(s), r) d s=\mathbb{E} \int_{t_{0}}^{t} e^{\gamma\left(s-t_{0}\right)}\left[\int_{\Omega}\left(u^{T}(s-\tau(s), x) u(s-\tau(s), x)+v^{T}(s-\tau(s), x) v(s-\tau(s), x)\right) d x\right] d s \\
& =\mathbb{E} \int_{t_{0}-\tau\left(t_{0}\right)}^{t-\tau(t)} e^{\gamma\left(\theta+\tau(s)-t_{0}\right)}\left[\int_{\Omega}\left(u^{T}(\theta, x) u(\theta, x)+v^{T}(\theta, x) v(\theta, x)\right) d x\right] \frac{d \theta}{1-\dot{\tau}(s)} \\
& \quad \leqslant h e^{\gamma \tau} \int_{t_{0}-\tau\left(t_{0}\right)}^{t} e^{\gamma\left(\theta-t_{0}\right)}\left[\int_{\Omega}\left(u^{T}(\theta, x) u(\theta, x)+v^{T}(\theta, x) v(\theta, x)\right) d x\right] d \theta \\
& \quad \leqslant h e^{\gamma \tau} \mathbb{E}\left\{\int_{t_{0}-\tau\left(t_{0}\right)}^{t_{0}} e^{\gamma\left(\theta-t_{0}\right)}\left[\int_{\Omega}\left(u^{T}(\theta, x) u(\theta, x)+v^{T}(\theta, x) v(\theta, x)\right) d x\right] d \theta+\frac{1}{\underline{\alpha}}\right. \\
& \left.\cdot \mathbb{E} \int_{t_{0}}^{t} e^{\gamma\left(s-t_{0}\right)}\left[\int_{\Omega}\left(u^{T}(s, x) P_{r} u(s, x)+v^{T}(s, x) P_{r} v(s, x)\right) d x\right] d s\right\} \\
& \leqslant h e^{\gamma \tau} \mathbb{E} \int_{t_{0}-\tau}^{t_{0}} e^{\gamma\left(\theta-t_{0}\right)}\left[\int_{\Omega}\left(u^{T}(\theta, x) u(\theta, x)+v^{T}(\theta, x) v(\theta, x)\right) d x\right] d \theta+\frac{h e^{\gamma \tau}}{\underline{\alpha}} \int_{t_{0}}^{t} \mathbb{E} V(s, r) d s \leqslant \frac{\tau h}{\gamma} \\
& \cdot e^{\gamma \tau} e^{\gamma\left(t_{0}-t_{0}\right)}\left(\mathbb{E}\|\varphi\|_{\tau}^{2}+\mathbb{E}\|\psi\|_{\tau}^{2}\right)+\frac{h e^{\gamma \tau}}{\underline{\alpha}} \int_{t_{0}}^{t} \mathbb{E} V(s, r) d s .
\end{aligned}
$$

It follows by (43) and (44) that, for $r \in S$,

$\mathbb{E} V(t, r)$

$$
\begin{aligned}
\leqslant & \left((1+\alpha) \mathbb{E} V\left(t_{0}, r\right)+\frac{\beta_{2} \tau h}{\gamma} e^{\gamma \tau}\left(\mathbb{E}\|\varphi\|_{\tau}^{2}+\mathbb{E}\|\psi\|_{\tau}^{2}\right)\right) \\
& +\left(\xi+\frac{\beta_{2} h e^{\gamma \tau}}{\underline{\alpha}}\right) \int_{t_{0}}^{t} \mathbb{E} V(s, r) d s \\
& +\alpha \sum_{t_{0}<t_{k}<t} \mathbb{E} V\left(t_{k}, r\right), \quad t \geqslant t_{0} .
\end{aligned}
$$

In addition, for any $t \geqslant t_{0}$, we may assume that $t \in\left(t_{k}, t_{k+1}\right]$. Since $\inf _{k=1,2, \ldots}\left(t_{k}-t_{k-1}\right) \geqslant \mu$, one can get

$$
(1+\alpha)^{k} \leqslant e^{\left(\left(t_{k}-t_{0}\right) / \mu\right) \ln (1+\alpha)} \leqslant e^{(\ln (1+\alpha) / \mu)\left(t-t_{0}\right)} .
$$

According to Lemma 4, we can conclude that $r \in S$,

$$
\begin{aligned}
& \mathbb{E} V(t, r) \leqslant\left((1+\alpha) \mathbb{E} V\left(t_{0}, r\right)\right. \\
& \left.+\frac{\beta_{2} \tau h}{\gamma} e^{\gamma \tau}\left(\mathbb{E}\|\varphi\|_{\tau}^{2}+\mathbb{E}\|\psi\|_{\tau}^{2}\right)\right)(1+\alpha)^{k}
\end{aligned}
$$

$$
\begin{aligned}
& \cdot e^{\left(\xi+\beta_{2} h e^{\gamma \tau} / \underline{\alpha}\right)\left(t-t_{0}\right)} \leqslant\left((1+\alpha) \bar{\alpha}+\frac{\beta_{2} \tau h}{\gamma} e^{\gamma \tau}\right) \\
& \cdot\left(\mathbb{E}\|\varphi\|_{\tau}^{2}+\mathbb{E}\|\psi\|_{\tau}^{2}\right) \\
& \cdot e^{\left(\ln (1+\alpha) / \mu+\xi+\beta_{2} h e^{\gamma \tau} / \underline{\alpha}\right)\left(t-t_{0}\right)}, \quad t \geqslant t_{0} .
\end{aligned}
$$

Moreover, we have

$$
\begin{aligned}
& \underline{\alpha}\left(\mathbb{E}\|u\|^{2}+\mathbb{E}\|v\|^{2}\right) e^{\gamma\left(t-t_{0}\right)} \leqslant \mathbb{E} V(t, r) \\
& \leqslant\left((1+\alpha) \bar{\alpha}+\frac{\beta_{2} \tau h}{\gamma} e^{\gamma \tau}\right)\left(\mathbb{E}\|\varphi\|_{\tau}^{2}+\mathbb{E}\|\psi\|_{\tau}^{2}\right) \\
& \cdot e^{\left(\ln (1+\alpha) / \mu+\xi+\beta_{2} h e^{\gamma \tau} / \underline{\alpha}\right)\left(t-t_{0}\right)}, \quad t \geqslant t_{0}
\end{aligned}
$$

which yields

$$
\begin{aligned}
& \mathbb{E}\|u\|^{2}+\mathbb{E}\|v\|^{2} \\
& \leqslant \frac{(1+\alpha) \bar{\alpha}+\left(\beta_{2} \tau h / \gamma\right) e^{\gamma \tau}}{\underline{\alpha}}\left(\mathbb{E}\|\varphi\|_{\tau}^{2}+\mathbb{E}\|\psi\|_{\tau}^{2}\right) \\
& \cdot e^{\left(\ln (1+\alpha) / \mu+\xi+\beta_{2} h e^{\gamma \tau} / \underline{\alpha}-\gamma\right)\left(t-t_{0}\right)}
\end{aligned}
$$


TABLE 1: Ratios between allowable upper bounds of time-delay $\tau$ (or impulse $\mathscr{C}$ ) and maximum of parameters $\mathscr{P}$.

\begin{tabular}{lccccc}
\hline & $\tau$ & $\mathscr{C}$ & $\mathscr{P}$ & $\tau / \mathscr{P}$ & $\mathscr{C} / \mathscr{P}$ \\
\hline Our Example 1 & 19 & 1.3 & 2.1 & 9.048 & 0.619 \\
{$[$ 24, Example] } & 0.5 & 1.35 & 2.16 & 0.2315 & 0.625 \\
{$[$ 25, Example 1] } & 4 & 1 & 3 & 1.333 & 0.333 \\
{$[16$, Example 1] } & 1 & 0.8 & 3.5 & 0.2857 & 0.2286 \\
\hline
\end{tabular}

$$
\begin{array}{r}
=\frac{(1+\alpha) \bar{\alpha}+\left(\beta_{2} \tau h / \gamma\right) e^{\gamma \tau}}{\alpha}\left(\mathbb{E}\|\varphi\|_{\tau}^{2}+\mathbb{E}\|\psi\|_{\tau}^{2}\right) \\
\cdot e^{-\left(\beta_{1} / \bar{\alpha}-\ln (1+\alpha) / \mu-\beta_{2} h e^{\gamma \tau} / \underline{\alpha}-\max _{r \in S}\left(\sum_{j \in S}^{j \neq r}\left(\pi_{r j} \bar{\alpha} / \underline{\alpha}\right)\right)\right)\left(t-t_{0}\right)}, \\
t \geqslant t_{0} .
\end{array}
$$

Moreover, it follows by (25) that

$$
\frac{\beta_{1}}{\bar{\alpha}}-\frac{\ln (1+\alpha)}{\mu}-\frac{\beta_{2} h e^{\gamma \tau}}{\underline{\alpha}}-\max _{r \in S}\left(\sum_{j \in S}^{j \neq r} \frac{\pi_{r j} \bar{\alpha}}{\underline{\alpha}}\right)>0 .
$$

According to Definition 3, the equilibrium point $u=0$ and $v=0$ of the problem (3)-(5) is globally stochastically exponential stability.

Remark 9. In [10, Theorem 3.1] and [9, Theorem 1], their impulsive conditions are similar as $C_{k r}^{T} P_{i} C_{k r}<P_{l}, \mathscr{C}_{k r}^{T} P_{i} \mathscr{C}_{k r}<$ $P_{l}$, for all $r, i, l \in S$. But in our Theorem 8 , the condition range on $C_{k r}$ and $\mathscr{C}_{k r}$ is enlarged owing to (18).

Remark 10. In [16, Theorem 3.1-3.2] and [36, Theorem 3.1$3.2]$, the conditions of all the stability criteria can only be verified via calculating by hand. However, we set up LMIbased criterion in our Theorem 8 by Schur Complement technique, which can be verified by computer Matlab LMI toolbox. In the following, Example 1 will offer a skillful method in a numerical example to illustrate more effectiveness and less conservativeness of our Theorem 8 . That is, the comprehensive application of computer Matlab LMI toolbox and the method of trial and error in Example 1 improves significantly the allowable upper bounds of time-delays and impulse (see Table 1 and Remark 11 for details).

\section{Numerical Example}

Example 1. Consider the following Markovian jumping BAM neural networks:

$$
\begin{aligned}
\frac{\partial u}{\partial t}= & \nabla \cdot(D(t, x, u) \circ \nabla u)-A_{r} u(t, x) \\
& +W_{1 r} f_{1}(v(t, x)) \\
& +W_{2 r} f_{2}(v(t-\tau(t), x)), \\
& x \in \Omega, t \neq t_{k},
\end{aligned}
$$

$$
\begin{aligned}
\frac{\partial v}{\partial t}= & \nabla \cdot(\mathscr{D}(t, x, v) \circ \nabla v)-B_{r} v(t, x) \\
& +\Gamma_{1 r} g_{1}(u(t, x)) \\
& +\Gamma_{2 r} g_{2}(u(t-\tau(t), x)), \\
& x \in \Omega, t \neq t_{k},
\end{aligned}
$$$$
\Delta u\left(t_{k}, x\right)=\left(C_{k}(r(t))-I\right) u\left(t_{k}, x\right),
$$$$
\Delta v\left(t_{k}, x\right)=\left(\mathscr{C}_{k}(r(t))-I\right) v\left(t_{k}, x\right),
$$$$
x \in \Omega, k=1,2, \ldots,
$$$$
u\left(s, x ; t_{0}, \varphi\right)=\varphi(s, x),
$$$$
v\left(s, x ; t_{0}, \psi\right)=\psi(s, x),
$$$$
t_{0}-\tau \leqslant s \leqslant t_{0}, x \in \Omega,
$$

$u\left(s, x ; t_{0}, \varphi\right)=0$

$v\left(s, x ; t_{0}, \varphi\right)=0$,

$$
x \in \partial \Omega, t \geqslant t_{0},
$$

where $u=\left(u_{1}, u_{2}\right)^{T}, v=\left(v_{1}, v_{2}\right)^{T} \in R^{2}, x=\left(x_{1}, x_{2}\right) \epsilon$ $(0,10) \times(0,10) \subset R^{2}$, and then $\lambda_{1}=0.02 \pi^{2}=0.1974[15$, Remark 2]:

$$
\begin{aligned}
D & =\left(\begin{array}{ll}
0.008 & 0.006 \\
0.007 & 0.005
\end{array}\right), \\
\mathscr{D} & =\left(\begin{array}{ll}
0.009 & 0.005 \\
0.007 & 0.006
\end{array}\right), \\
H_{1} & =\left(\begin{array}{cc}
0.3 & 0 \\
0 & 0.4
\end{array}\right), \\
H_{2} & =\left(\begin{array}{cc}
0.4 & 0 \\
0 & 0.5
\end{array}\right), \\
\mathscr{H}_{1} & =\left(\begin{array}{cc}
0.5 & 0 \\
0 & 0.4
\end{array}\right), \\
\mathscr{H}_{2} & =\left(\begin{array}{cc}
0.4 & 0 \\
0 & 0.3
\end{array}\right) .
\end{aligned}
$$

From the above data, we know that $d=0.005$ and $\tilde{d}=0.005$. Consider two modes for the Markovian jumping impulsive system (51). For mode 1,

$$
\begin{aligned}
& A_{1}=\left(\begin{array}{cc}
0.9 & 0 \\
0 & 1.2
\end{array}\right), \\
& B_{1}=\left(\begin{array}{cc}
2 & 0 \\
0 & 1.8
\end{array}\right),
\end{aligned}
$$




$$
\begin{aligned}
W_{11} & =\left(\begin{array}{cc}
0.11 & 0.003 \\
0.003 & 0.12
\end{array}\right), \\
W_{21} & =\left(\begin{array}{ll}
0.13 & 0.002 \\
0.002 & 0.12
\end{array}\right), \\
\Gamma_{11} & =\left(\begin{array}{ll}
0.7 & 0.4 \\
0.3 & 0.8
\end{array}\right), \\
\Gamma_{21} & =\left(\begin{array}{cc}
0.8 & 0.3 \\
0.5 & 0.6
\end{array}\right), \\
C_{k 1} & =\left(\begin{array}{cc}
1.9 & 0 \\
0 & 1.7
\end{array}\right), \\
\mathscr{C}_{k 1} & =\left(\begin{array}{cc}
1.8 & 0 \\
0 & 1.6
\end{array}\right) .
\end{aligned}
$$

For mode 2,

$$
\begin{aligned}
A_{2} & =\left(\begin{array}{cc}
1.2 & 0 \\
0 & 1.3
\end{array}\right), \\
B_{2} & =\left(\begin{array}{ll}
2.1 & 0 \\
0 & 2
\end{array}\right), \\
W_{12} & =\left(\begin{array}{ll}
0.12 & 0.003 \\
0.003 & 0.11
\end{array}\right), \\
W_{22} & =\left(\begin{array}{cc}
0.11 & 0.002 \\
0.002 & 0.12
\end{array}\right), \\
\Gamma_{12} & =\left(\begin{array}{cc}
0.13 & 0.003 \\
0.003 & 0.16
\end{array}\right), \\
\mathscr{C}_{k 2} & =\left(\begin{array}{ll}
2 & 0 \\
0 & 1.7
\end{array}\right) . \\
\Gamma_{22} & =\left(\begin{array}{cc}
0.17 & 0.002 \\
0.002 & 0.13
\end{array}\right), \\
C_{k 2} & =\left(\begin{array}{cc}
2.3 & 0 \\
0 & 1.9
\end{array}\right),
\end{aligned}
$$

Let $\pi_{11}=-0.6, \pi_{12}=0.6, \pi_{21}=0.2$, and $\pi_{22}=-0.2$. From the above data, we can select $\alpha=4.3$. Then it is not difficult to verify that condition (18) holds for any mode $r \in S=\{1,2\}$. In addition, fix $\mu=3.5, \tau=19, h=1.2$, and $\gamma=0.001$. Now we use the Matlab LMI toolbox to solve the linear matrices inequalities (19)-(24) and then the feasibility data follows:

$$
\begin{aligned}
& P_{1}=\left(\begin{array}{cc}
12.3228 & 0 \\
0 & 12.1417
\end{array}\right), \\
& P_{2}=\left(\begin{array}{cc}
12.1357 & 0 \\
0 & 12.1246
\end{array}\right), \\
& \underline{\alpha}=11.3765, \\
& \bar{\alpha}=13.1353, \\
& \beta_{1}=16.8064, \\
& \beta_{2}=0.4689 .
\end{aligned}
$$

Moreover, substituting the above data into (25) results in

$$
\begin{aligned}
& \frac{\beta_{1}}{\bar{\alpha}}-\frac{\ln (1+\alpha)}{\mu}-\sum_{j \in S}^{j \neq 1} \frac{\pi_{1 j} \bar{\alpha}}{\underline{\alpha}}-\frac{\beta_{2} h e^{\gamma \tau}}{\underline{\alpha}}=0.0598>0, \\
& \frac{\beta_{1}}{\bar{\alpha}}-\frac{\ln (1+\alpha)}{\mu}-\sum_{j \in S}^{j \neq 2} \frac{\pi_{2 j} \bar{\alpha}}{\underline{\alpha}}-\frac{\beta_{2} h e^{\gamma \tau}}{\underline{\alpha}}=0.5217>0,
\end{aligned}
$$

which implies that all the assumptions of Theorem 8 are satisfied. And then the equilibrium point $u=0$ and $v=0$ of system (51) is globally stochastically exponential stability. On the whole, Example 1 illustrates more effectiveness and less conservativeness of our Theorem 8 than other related literatures (see Table 1 for details).

Remark 11. In Example 1, to verify the condition (25), we need to employ comprehensively the computer Matlab LMI toolbox and the method of trial and error. To compare the upper bounds of time-delay and impulse in various related literature, we need to compute and compare the ratios between the maximum of allowable delay (or impulsive) and maximum of parameters in various related literature, because the maximum of allowable delay may rise as parameters of numerical examples become bigger. From Table 1, the ratios of our Example 1 are bigger than those of $[24,25]$ to some extent. And hence, there are some advantages of the utilization of Gronwall-Bellman-type inequality compared to that of other inequalities in $[24,25]$. Moreover, LMIbased criterion in our Theorem 8 is more effective than handcomputation based criteria of $[16,36]$.

Remark 12. From the above data in Example 1, it is obvious that condition (18) in Theorem 8 holds. But the similar impulsive conditions $C_{k r}^{T} P_{i} C_{k r}<P_{l}, \mathscr{C}_{k r}^{T} P_{i} \mathscr{C}_{k r}<P_{l}$ for all $r, i, l \in$ $S$ in [10, Theorem 3.1] and [9, Theorem 1] can not be satisfied. Indeed,

$$
\begin{aligned}
\mathscr{C}_{k 2}^{T} P_{1} \mathscr{C}_{k 2} & =\left(\begin{array}{cc}
2 & 0 \\
0 & 1.7
\end{array}\right)\left(\begin{array}{cc}
12.3228 & 0 \\
0 & 12.1417
\end{array}\right)\left(\begin{array}{cc}
2 & 0 \\
0 & 1.7
\end{array}\right) \\
& =\left(\begin{array}{cc}
49.2912 & 0 \\
0 & 35.0895
\end{array}\right)>P_{r}, \quad r=1,2 .
\end{aligned}
$$


This illustrates that the stability criterion of our Theorem 8 can judge what [10, Theorem 3.1] and [9, Theorem 1] can not do, which shows clearly that the allowable upper bounds of impulse are improved significantly against those of [10, Theorem 3.1], [9, Theorem 1], [15, Theorem 1], [3, Theorem 4.1], [4, Theorem 3.2], and [19, Theorem 3.2] owing to employing the Gronwall-Bellman-type impulsive integral inequality.

\section{Conclusions}

In this paper, Schur Complement technique and GronwallBellman-type impulsive integral inequality are synthetically applied to stability analysis of impulsive delayed Markovian jumping reaction-diffusion bidirectional associative memory (BAM) neural networks, and LMI-based exponential stability criterion is obtained. It is the first time to obtain the LMIbased stability criterion for BAM neural networks via Gronwall-Bellman-type impulsive integral inequality. The main result of this paper improves the allowable upper bounds of impulse in [10, Theorem 3.1], [9, Theorem 1], [15, Theorem 1], [3, Theorem 4.1], [4, Theorem 3.2], and [19, Theorem 3.2]. Finally, a numerical example in Section 4 is presented to illustrate the effectiveness of the proposed method (see Remarks 10 and 11).

\section{Conflict of Interests}

All the authors declare that there is no conflict of interests regarding the publication of this paper.

\section{Authors' Contribution}

Ruofeng Rao wrote the original paper, and Shouming Zhong examined the original paper in detail before submission. Xiongrui Wang is in charge of the correspondence.

\section{Acknowledgments}

The authors would like to thank the reviewers and the editors for their valuable suggestions and comments which have led to a much improved paper. This work is supported by Scientific Research Fund of Science Technology Department of Sichuan Province (2012JYZ010) and by Scientific Research Fund of Sichuan Provincial Education Department (14ZA0274 and 12ZB349).

\section{References}

[1] B. Kosko, "Adaptive bidirectional associative memories," Applied Optics, vol. 26, no. 3, pp. 4947-4960, 1987.

[2] L. Wan and Q. Zhou, "Global exponential stability of BAM neural networks with time-varying delays and diffusion terms," Physics Letters, Section A: General, Atomic and Solid State Physics, vol. 371, no. 1-2, pp. 83-89, 2007.

[3] Q. Song and J. Cao, "Exponential stability for impulsive BAM neural networks with time-varying delays and reaction-diffusion terms," Advances in Difference Equations, vol. 2007, Article ID 78160, 18 pages, 2007.
[4] R. Rao, S. Zhong, and X. Wang, "Stochastic stability criteria with LMI conditions for Markovian jumping impulsive BAM neural networks with mode-dependent time-varying delays and nonlinear reaction-diffusion," Communications in Nonlinear Science and Numerical Simulation, vol. 19, no. 1, pp. 258-273, 2014.

[5] W. Zhang, J. Li, and M. Chen, "Global exponential stability and existence of periodic solutions for delayed reaction-diffusion BAM neural networks with Dirichlet boundary conditions," Boundary Value Problems, vol. 2013, article 105, 2013.

[6] W. Zhang and J. Li, "Global exponential synchronization of delayed BAM neural networks with reaction-diffusion terms and the Neumann boundary conditions," Boundary Value Problems, vol. 2012, article 2, 2012.

[7] L. Wang, Z. Zhang, and Y. Wang, "Stochastic exponential stability of the delayed reaction-diffusion recurrent neural networks with Markovian jumping parameters," Physics Letters A, vol. 372, no. 18, pp. 3201-3209, 2008.

[8] V. Lakshmikantham, D. D. Bainov, and P. S. Simeonov, Theory of Impulsive Differential Equations, World Scientific Publishing, Singapore, 1989.

[9] M. Dong, H. Zhang, and Y. Wang, "Dynamics analysis of impulsive stochastic Cohen-Grossberg neural networks with Markovian jumping and mixed time delays," Neurocomputing, vol. 72, no. 7-9, pp. 1999-2004, 2009.

[10] R. Rakkiyappan and P. Balasubramaniam, "Dynamic analysis of Markovian jumping impulsive stochastic Cohen-Grossberg neural networks with discrete interval and distributed timevarying delays," Nonlinear Analysis: Hybrid Systems, vol. 3, no. 4, pp. 408-417, 2009.

[11] J. Cheng, H. Zhu, Y. Ding, Z. Zhong, and Q. Zhong, "Stochastic finite-time boundedness for Markovian jumping neural networks with time-varying delays," Applied Mathematics and Computation, vol. 242, pp. 281-295, 2014.

[12] C. Yuan and J. Lygeros, "Stabilization of a class of stochastic differential equations with Markovian switching," Systems and Control Letters, vol. 54, no. 9, pp. 819-833, 2005.

[13] J. Tian, Y. Li, J. Zhao, and S. Zhong, "Delay-dependent stochastic stability criteria for Markovian jumping neural networks with mode-dependent time-varying delays and partially known transition rates," Applied Mathematics and Computation, vol. 218, no. 9, pp. 5769-5781, 2012.

[14] Q. Zhu and J. Cao, "Stochastic stability of neural networks with both Markovian jump parameters and continuously distributed delays," Discrete Dynamics in Nature and Society, vol. 2009, Article ID 490515, 20 pages, 2009.

[15] J. Pan and S. Zhong, "Dynamical behaviors of impulsive reaction-diffusion Cohen-Grossberg neural network with delays," Neurocomputing, vol. 73, no. 7-9, pp. 1344-1351, 2010.

[16] Y. Zhang and Q. Luo, "Global exponential stability of impulsive delayed reaction-diffusion neural networks via Hardy-Poincarè inequality," Neurocomputing, vol. 83, no. 15, pp. 198-204, 2012.

[17] J. Pan, X. Liu, and S. Zhong, "Stability criteria for impulsive reaction-diffusion Cohen-Grossberg neural networks with timevarying delays," Mathematical and Computer Modelling, vol. 51, no. 9-10, pp. 1037-1050, 2010.

[18] X. Li, J. Zou, and E. Zhu, "pth moment exponential stability of impulsive stochastic neural networks with mixed delays," Mathematical Problems in Engineering, vol. 2012, Article ID 175934, 20 pages, 2012. 
[19] R. Rao, "Delay-dependent exponential stability for nonlinear reaction-diffusion uncertain Cohen-Grossberg neural networks with partially known transition rates via Hardy-Poincaré inequality," Chinese Annals of Mathematics. Series B, vol. 35, no. 4, pp. 575-598, 2014.

[20] Q. Feng and B. Zheng, "Generalized Gronwall-Bellman-type delay dynamic inequalities on time scales and their applications," Applied Mathematics and Computation, vol. 218, no. 15, pp. 7880-7892, 2012.

[21] I. N’Doye, M. Zasadzinski, M. Darouach, N.-E. Radhy, and A. Bouaziz, "Exponential stabilization of a class of nonlinear systems: a generalized Gronwall-Bellman lemma approach," Nonlinear Analysis: Theory, Methods \& Applications, vol. 74, no. 18, pp. 7333-7341, 2011.

[22] Q. Feng, F. Meng, and B. Zheng, "Gronwall-Bellman type nonlinear delay integral inequalities on time scales," Journal of Mathematical Analysis and Applications, vol.382, no. 2, pp. 772784, 2011.

[23] A. Alofi, J. Cao, A. Elaiw, and A. Al-Mazrooei, "Delaydependent stability criterion of caputo fractional neural networks with distributed delay," Discrete Dynamics in Nature and Society, vol. 2014, Article ID 529358, 6 pages, 2014.

[24] X. Zhang, S. Wu, and K. Li, "Delay-dependent exponential stability for impulsive Cohen-Grossberg neural networks with time-varying delays and reaction-diffusion terms," Communications in Nonlinear Science and Numerical Simulation, vol. 16, no. 3, pp. 1524-1532, 2011.

[25] K. Li and Q. Song, "Exponential stability of impulsive CohenGrossberg neural networks with time-varying delays and reaction-diffusion terms," Neurocomputing, vol. 72, no. 1-3, pp. 231240, 2008.

[26] B. Kosko, "Bidirectional associative memories," IEEE Transactions on Systems, Man, and Cybernetics, vol. 18, no. 1, pp. 49-60, 1988.

[27] C. Qi, C. Li, and T. Huang, "Stability of inertial BAM neural network with time-varying delay via impulsive control," Neurocomputing, vol. 161, pp. 162-167, 2015.

[28] K. Mathiyalagan, J. H. Park, and R. Sakthivel, "Synchronization for delayed memristive BAM neural networks using impulsive control with random nonlinearities," Applied Mathematics and Computation, vol. 259, pp. 967-979, 2015.

[29] G. Sun and Y. Zhang, "Exponential stability of impulsive discrete-time stochastic BAM neural networks with timevarying delay," Neurocomputing, vol. 131, pp. 323-330, 2014.

[30] Q. Zhu, R. Rakkiyappan, and A. Chandrasekar, "Stochastic stability of Markovian jump BAM neural networks with leakage delays and impulse control," Neurocomputing, vol. 136, pp. 136151, 2014.

[31] L. Pan and J. Cao, "Stability of bidirectional associative memory neural networks with Markov switching via ergodic method and the law of large numbers," Neurocomputing, 2015.

[32] V. Maz'ya, Sobolev Spaces: with Applications to Elliptic Partial Differential Equations, vol. 342 of Grundlehren der Mathematischen Wissenschaften [Fundamental Principles of Mathematical Sciences, Springer, Berline, Germany, 2nd edition, 2011.

[33] P. Lindqvise, "On the Equation $\operatorname{div}(|\nabla u| p-2 \nabla u)+\lambda|u| p-2 u=$ 0," Proceedings of the American Mathematical Society, vol. 109, pp. 159-164, 1990.

[34] Z. Wang, Y. Liu, L. Yu, and X. Liu, "Exponential stability of delayed recurrent neural networks with Markovian jumping parameters," Physics Letters A, vol. 356, no. 4-5, pp. 346-352, 2006.

[35] S. P. Boyd, L. F. Ghaoui, F. Feron, and V. Balakrishnan, Linear Matrix Inequalities in Systems and Control Theory, SIAM, Philadelphia, Pa, USA, 1994.

[36] Y. Zhang and Q. Luo, "Novel stability criteria for impulsive delayed reaction-diffusion Cohen-Grossberg neural networks via Hardy-Poincarè inequality," Chaos, Solitons \& Fractals, vol. 45, no. 8, pp. 1033-1040, 2012. 


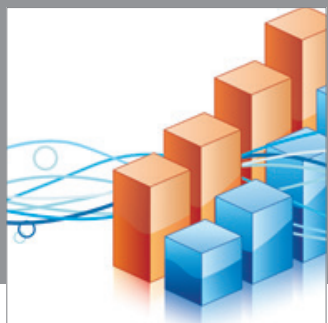

Advances in

Operations Research

mansans

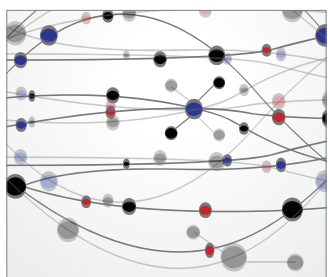

The Scientific World Journal
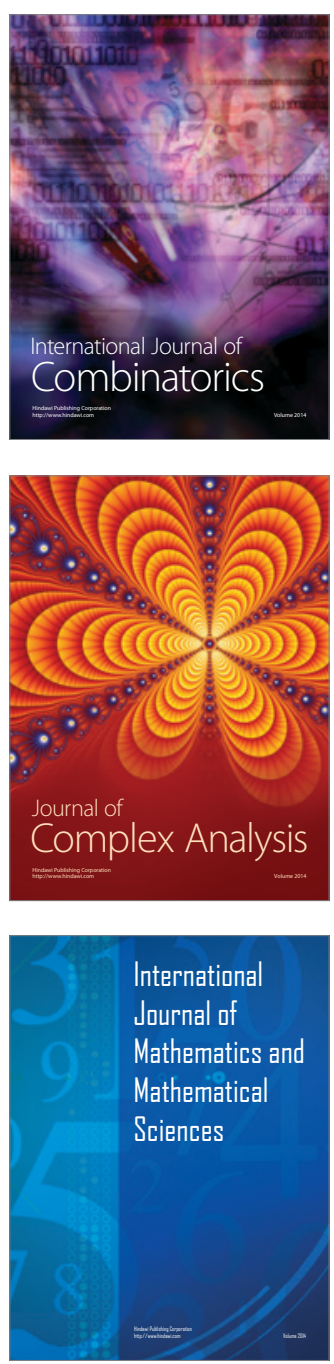
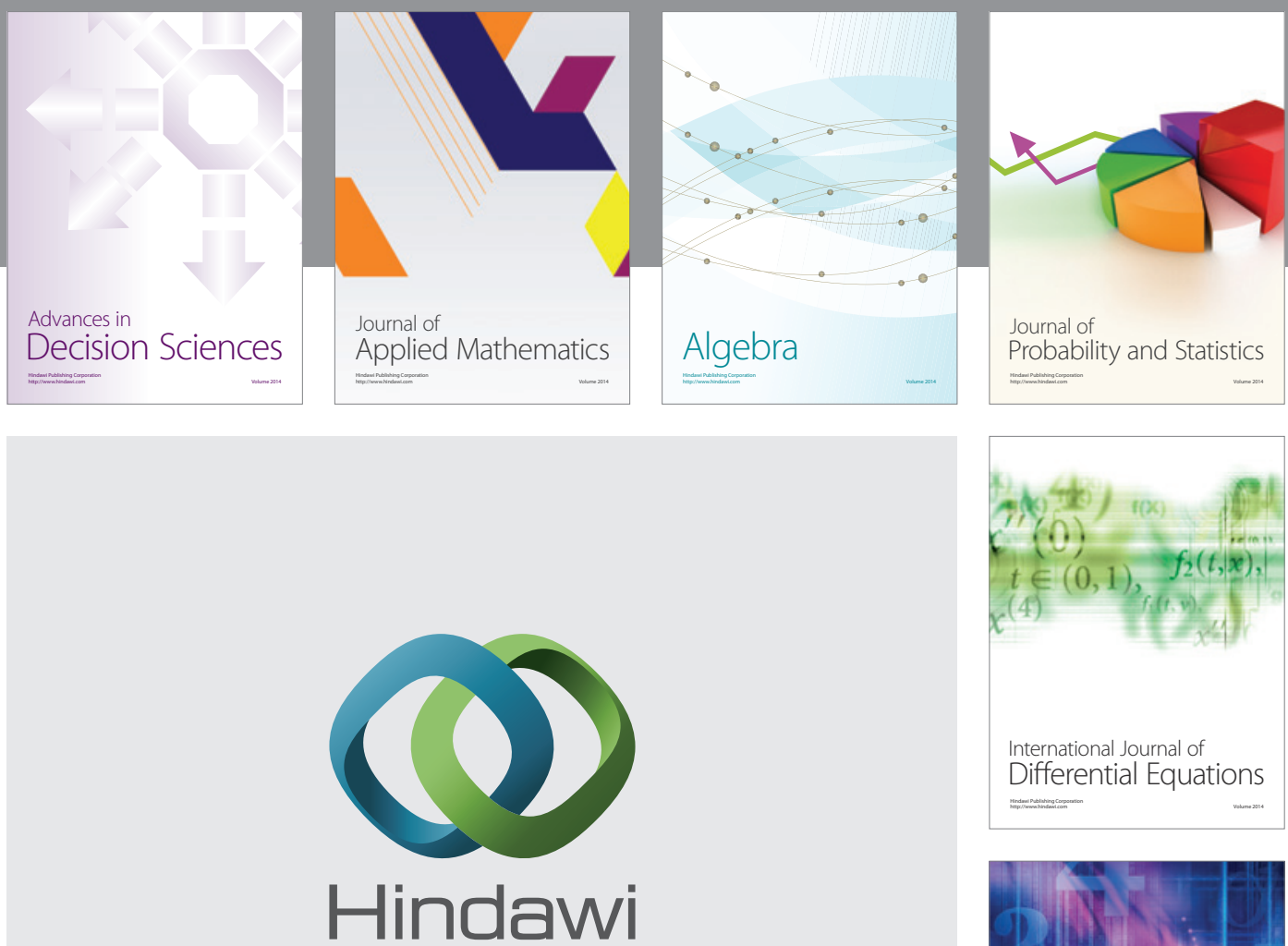

Submit your manuscripts at http://www.hindawi.com
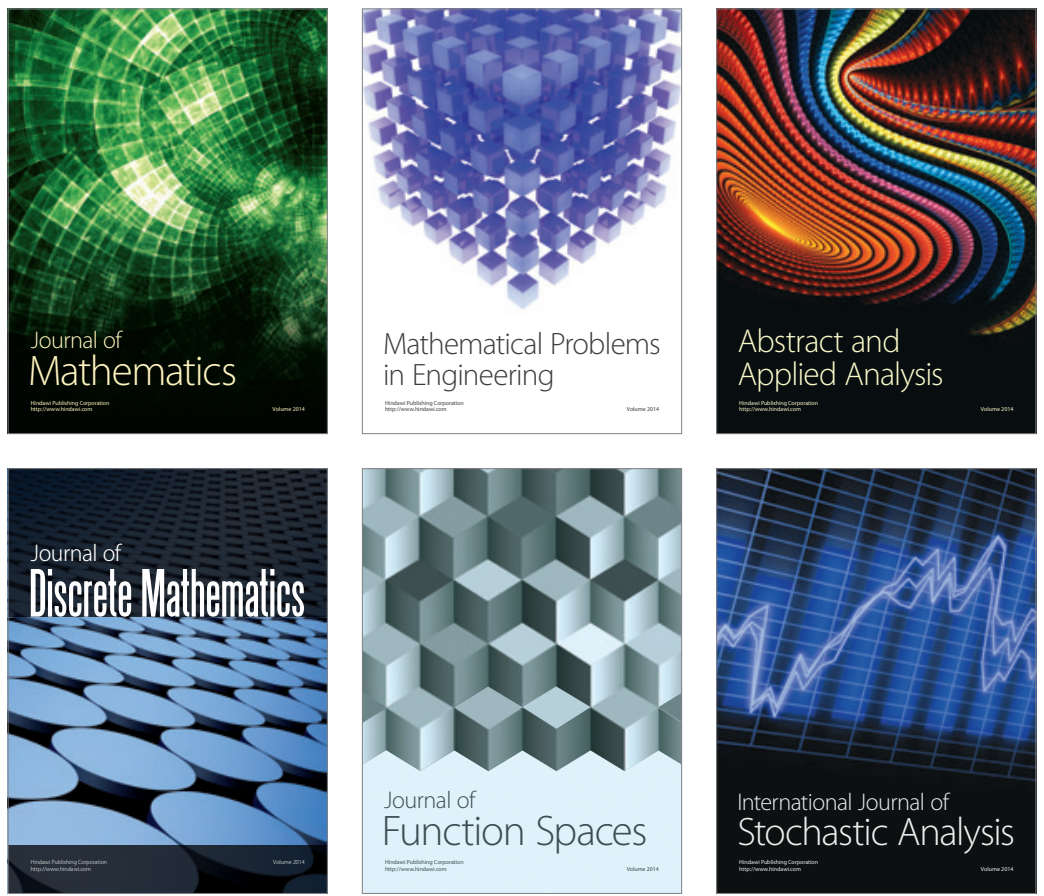

Journal of

Function Spaces

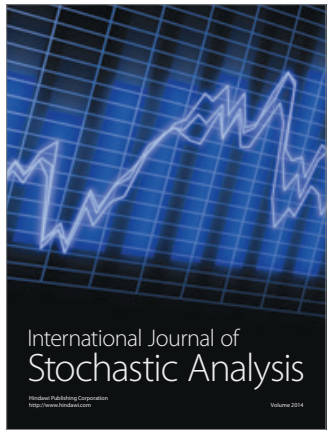

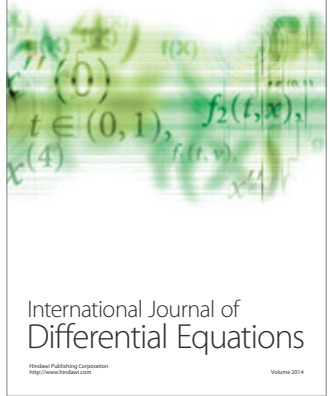
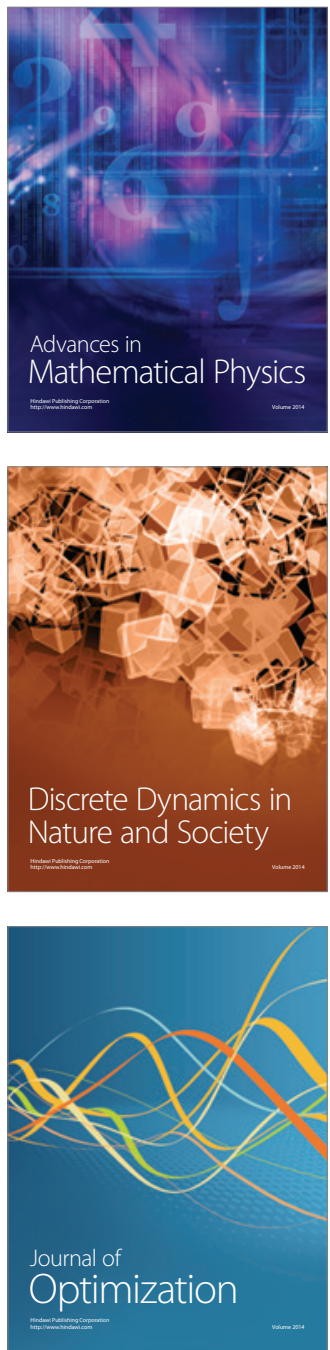